\title{
Interactive comment on "Assimilating Shallow Soil Moisture Observations into Land Models with a Water Budget Constraint" by Bo Dan et al.
}

\section{Anonymous Referee \#2}

Received and published: 2 April 2020

The paper titled "Assimilating Shallow Soil Moisture Observations into Land Models with a Water Budget Constraint" presented several modifications to the EnKF data assimilation (DA) that potentially improve DA performance in soil moisture (SM) modeling using shallow-layer observations. A forecast error covariance matrix inflation approach to avoid filter divergence due to underestimated sampling and modeling errors is shown to improve modeling accuracy of SM in layers close to the observation, but leads to increased error in the deeper layers. A vertical localization method is applied to limit the updates to the deep layers to mitigate the errors introduced in the deeper layers. A weak constraint on water balance is able to reduce the water balance residual which is increased due to the forecast error covariance inflation at the price of small increase in the analysis error. Overall the results indicate potential usefulness of such

Printer-friendly version

Discussion paper 
modifications in improving soil moisture assimilation accuracy of surface soil moisture observations.

However, there is a major issue in the experiment design the raises my concern, i.e. the lack of observation bias-correction. I found the authors' reasoning behind adopting the "traditional bias-blind data assimilation framework" (line 112-117) unconvincing, as there is no evidence to support the "observations" are unbiased relative to the model background in both the synthetic and real-data experiments in this study. Also, it is wellknown that remotely sensed soil moisture (the intended application of the proposed modifications) and modeled soil moisture often exhibit different dynamic ranges which warrants the use of a "bias-aware" approach instead (see e.g. Kumar et al. 2012, doi:10.1029/2010WR010261).

In addition, there is an apparent misunderstanding of the Koster et al. (2009) and Reichle and Koster (2004) works where the authors stated that "A major objective of soil moisture data assimilation is to address biases in models and observations" (line 110-111). In fact, both publications indicated the importance of removing the bias in the statistical moments in the observations relative to the model background prior to data assimilation. The major objective of data assimilation is not to remove the bias in model states but to reduce the random, mean-zero noise in the model states, with the model state climatology respected. Even if the observations are considered unbiased, it is recommended that the observations be "scaled" to match the statistical moments of the model states (with long enough time-series). It is well known that directly assimilation of raw observations likely causes model integration to drift, i.e. introduce further bias to the model states. Therefore, the model water balance residual after the soil moisture update in the experiments in this study may be partly attributed to assimilating observations without bias-correction (relative to model), and the true effect of the weak water balance constraint is not accurately revealed.

I would like suggest that the DA experiments repeated with a more robust "bias-aware" approach, to rule out the impact of observation bias in the analysis errors so that the

Printer-friendly version

Discussion paper

\section{2}


effects of the proposed modifications are better isolated.

Other minor comments: (line) 65-74: irrelevant to the topic of the paper and should be removed. 229: why directly update canopy water content and snow water equivalent when these two variables are not regulated by near-surface soil moisture? 448: remove "the" following "cover" 478: "the different experiments" $\rightarrow$ "different cases" 479-480: for better understanding of the magnitude of improvement, use a percentage scale for water balance residuals 494 "deflation «of» the water balance ..."; "plain" ->"plainly" 511 "shreshould" ->"threshold"

Sec 7.2 Again, one should be careful to use data assimilation to achieve "bias correction" in model states. This is another example of misunderstanding the major objective of DA in this work. Seemingly reduced systematic bias in modeled soil moisture may be an artifact due to biased observation relative to model background.

Interactive comment on Hydrol. Earth Syst. Sci. Discuss., https://doi.org/10.5194/hess-2019696, 2020. 